\title{
Flocculation by Cationic Polyelectrolytes: Relating Efficiency with Polyelectrolyte Characteristics
}

\author{
M. G. Rasteiro, ${ }^{1}$ F. A. P. Garcia, ${ }^{1}$ P. J. Ferreira, ${ }^{1}$ E. Antunes, ${ }^{1}$ D. Hunkeler, ${ }^{2}$ C. Wandrey ${ }^{3}$ \\ ${ }^{1}$ Chemical Engineering Department, Research Centre for Chemical Processes Engineering and Forest Products, \\ Coimbra, Portugal \\ ${ }_{3}^{2}$ AQUA+TECH, La Plaine, Geneva, Switzerland \\ ${ }^{3}$ Institut des Sciences et ingénierie chimiques, Chaire Merck-Serono en technologies d'administration de médicaments, \\ Ecole Polytechnique Fédérale de Lausanne, Switzerland
}

Received 11 February 2009; accepted 3 December 2009

DOI 10.1002/app.31903

Published online 22 February 2010 in Wiley InterScience (www.interscience.wiley.com).

\begin{abstract}
A series of acrylamide/dimethylaminoethylacrylate methyl chloride copolymers (AM-co-DMAEA) was evaluated as flocculants for model Precipitated Calcium Carbonate (PCC) in distilled water. These cationic polyelectrolytes (PEL) differed by their AM/DMAEA ratio, i.e., their charge density, chain architecture (linear and branched), their intrinsic viscosity (IV), and zeta potential of their aqueous solutions. The IV being directly related to the hydrodynamic volume of the PEL and the zeta potential reflecting the effective charges in suspension were selected for practically useful correlations with flocculation performance. The fractal dimension $\left(d_{F}\right)$ and the scattering exponent (SE) of the flocs, analyzed by the laser diffraction method, were taken as indication of primary particle and aggregate assem-
\end{abstract}

bly. For the optimum PEL dosage, SE and $d_{F}$ varied with the IV and zeta potential. It was observable that floc size, floc resistance, and floc reorganization correlate with the PEL characteristics. Copolymers with lower IV, which is here associated with PEL of similar molar mass but lower charge density, yielded larger and less resistant flocs. Copolymers with higher IV originated smaller more compact flocs being more resistant. In high shear situations, where floc breakage should be avoided, as it is the case in papermaking, polymers with high IV yielding large SE are advantageous. ( 2010 Wiley Periodicals, Inc. J Appl Polym Sci 116: 3603-3612, 2010

Key words: copolymerization; water-soluble polymers; polyelectrolytes; flocculation; laser diffraction (LDS)

\section{INTRODUCTION}

Synthetic polyelectrolytes (PEL) based on acrylamide (AM) are produced industrially worldwide. They are mostly copolymers of AM with either anionic or cationic monomers or result from polymer modification. In particular, cationic PEL have found widespread industrial application as flocculants in branches of industry such as mining, water resource management, and papermaking. Chemically, these PEL are a group of macromolecules where the charges are localized pendant to the hydrophobic carbon backbone, most frequently as quaternary ammonium substituents, and which have molar masses in the order of $10^{6}$ to $10^{7} \mathrm{~g} / \mathrm{mol}$. Occasionally, nonlinear/branched flocculants are reported to have improved performance compared to their linear analogous. ${ }^{1}$

Charged macromolecules with high molar masses, in particular technical products, can pose characterization difficulties. Sample purification, necessary for techniques such as light scattering, can be difficult

Correspondence to: M. G. Rasteiro (mgr@eq.uc.pt).

Journal of Applied Polymer Science, Vol. 116, 3603-3612 (2010)

(C) 2010 Wiley Periodicals, Inc. leaving impurities of similar sizes to the coils to interact with the radiation. Chromatographic separations are inherently based on multiple modes and the interpretation can be imprecise. Further, stationary phases possess exclusion limits too low to characterize the full distribution of very high molar mass charged water-soluble polymers. A reliable method to characterize such types of PEL tends to be by analytical ultracentrifugation combined with dilution viscometry as reported previously. ${ }^{2,3}$ The intrinsic viscosity (IV) is a suitable parameter, in particular, if it is the intention to compare the impact of PEL of different chain architecture and charge density/ chemical structure on technological processes such as flocculation, where the polymer coil dimensions are important. The prerequisites, however, are known and constant environment/solution conditions as set in this study.

For a known molar mass, the polymer coil size in solution depends on the chemical structure, chain architecture (linear, branched), and the solvent quality. Whereas the coil expansion for neutral polymers is limited even in good solvents, the chain expansion of PEL is theoretically unlimited and a function of the ionic strength governing the Debye length. Therefore, the knowledge of the molar mass cannot be considered as a sufficient parameter for studying 
flocculation. It is worth mentioning that the ionic strength of the industrial water, for example present in papermaking, is usually too low to ensure conditions where the PEL approaches a fictive electroneutrality.

Flocculation can best be understood and modeled if any experimental work is based on a series of well-defined standards. ${ }^{4}$ These would, ideally, vary with respect to one property, such as polymer coil size, charge density or, for nonlinear polymers, the degree of branching. The latter is included as recent Monte Carlo simulations have indicated that completely soluble PEL can be formed with higher degrees of nonlinearity than previously envisioned based on experimental evidence alone. ${ }^{5}$ Such studies which use model macromolecules in a systematic set of flocculation experiments are rare and this has motivated this investigation. Understanding the PEL-particle interactions, so essential in quantifying flocculation, challenged us to synthesize a series of AM/dimethylaminoethylacrylate methyl chloride copolymers (AM-co-DMAEA), which differ by their AM/DMAEA ratio, i.e., their charge density, chain architecture, i.e., linear and branched, using a previously optimized synthesis process. ${ }^{3,6-8}$

Many studies demonstrated that, depending on the flocculant used, aggregation of the particles can occur by charge neutralization, patching, or bridging. ${ }^{9-15}$ Aggregation by charge neutralization occurs due to reduction of the repulsive forces between particles by adding an electrolyte or a very low molar mass PEL. The optimal dosage corresponds to the isoelectric point. ${ }^{12,16}$ The patch model is based on the formation of cationic sites or "patches" of the cationic polyelectrolyte on the negative surface of the particle. Flocculation will then take place by electrostatic attraction between the oppositely charged sites on the particles. ${ }^{10}$ Flocculation of particles induced by PEL of very high molar mass occurs by the bridging mechanism. This mechanism was firstly proposed by La Mer and Healy. ${ }^{17}$ Fleer and Scheutjens described the bridging mechanism as the result of three consecutive steps: polymer adsorption, bridging, and depletion. ${ }^{18}$ The conformation of the polymer at the particle surface and, thus, the bridging performance, depends on the polymer characteristics. $9,12,15,19$ As the polymer charge density increases, the bridging capability is reduced because there is a tendency for the polymer chains to adopt a flat conformation on the particle surface, which results in the formation of cationic patches that attract the PEL-free surfaces of other particles. ${ }^{20,21} \mathrm{In}$ this case, the adsorption rate decreases and the reconformation occurs faster as the charge of the PEL increases. Moreover, the flocculant concentration also affects the reconformation rate: polymer rearrangement is relatively fast at low surface con- centration but rather slowly on crowded surfaces, as neighboring molecules interfere with the rearrangement. ${ }^{13,22}$

As flocculation affects the product quality and economy of many industrial processes, it is important knowing, for each flocculant, the predominant interactions and mechanisms to optimize the flocculation process.

Advances in the quantification of the flocculation process include the application of the laser diffraction method (LDS) in the laboratory ${ }^{13,23,24}$ and in situations closer to industrial application such as in papermaking. ${ }^{25}$ This investigation uses the LDS method, which was adapted previously by the authors to monitor flocculation processes continuously, ${ }^{13,23}$ to systematically study the influence of the PEL characteristics on the mechanism of flocculation, on the size and density of the flocs formed, the kinetics of formation, and the optimal flocculant dosage. The floc density is well represented by the fractal dimension, $d_{F}{ }^{26}$ or the scattering exponent, SE. ${ }^{27}$ Previously, the authors presented and discussed the flocculation kinetic curves for some cationic polyacrylamides used to flocculate model Precipitated Calcium Carbonate (PCC) particles. ${ }^{26,28}$ In this study, to better understand the influence of the PEL properties on the likely flocculation mechanisms and, thereafter, on the characteristics of the flocs produced and of the flocculation process itself, we have related size, resistance, and density of the flocs as well as flocs reorganization and flocculant dosage required, with the IV and zeta potential, while keeping constant the model particles. In this way, it was possible to find some trends regarding the relation between flocs and PEL characteristics.

\section{EXPERIMENTAL}

\section{Polymer synthesis}

The copolymers were synthesized according to patented principles. ${ }^{6-8}$ Subsequently, specific details of the synthesis of the copolymers used here are described.

(AM-co-DMAEA) was prepared by inverse-emulsion polymerization. Polymerizations were carried out with an aqueous to organic phase ratio of 0.737 : $0.263(\mathrm{wt} / \mathrm{wt})$ in a 1-L glass reactor. The total mass of the reacting mixture was $500 \mathrm{~g}$. Before reaction, the aqueous phase was added to the organic phase under mixing $(675 \mathrm{rpm}$ with an overhead agitation with a pitched blade impeller). During pre-emulsification, the mixture was degassed with nitrogen (>99.99\%, Carbagas, Geneva) until the residual oxygen level was below $2 \mathrm{ppm}$. The degassing step normally required 60-90 min. 
TABLE I

Polymer Composition and Characteristics

\begin{tabular}{lccccc}
\hline \multicolumn{1}{c}{ PEL } & $\begin{array}{c}\text { DMAEA } \\
(\text { wt \% })\end{array}$ & $\begin{array}{c}\text { DMAEA } \\
(\mathrm{mol} \%)\end{array}$ & $\begin{array}{c}\text { Charge content } \\
(\mathrm{meq} / \mathrm{g})\end{array}$ & $\begin{array}{c}\text { Linear charge } \\
\text { distance }(\mathrm{nm})\end{array}$ & $\begin{array}{c}\mathrm{IV}^{\mathrm{a}} \\
(\mathrm{mL} / \mathrm{g})\end{array}$ \\
\hline BHMW & 80 & 59.5 & 4.13 & 0.42 & 3050 \\
E1 & 50 & 27 & 2.58 & 0.93 & 2508 \\
E1 & 50 & 27 & 2.58 & & 2720 \\
E1++++ & 50 & 27 & 2.58 & 3.0 & 1510 \\
G1 & 20 & 8.4 & 1.03 & & 1210 \\
G1 & 20 & 8.4 & 1.03 & & 1190 \\
G1 +++ & 20 & 8.4 & 1.03 & & \\
\hline
\end{tabular}

${ }^{\text {a }}$ Intrinsic viscosity in $0.05 \mathrm{M} \mathrm{NaCl}$ calculated according to Huggins. ${ }^{2}$

Polymerizations were initiated at $19-21^{\circ} \mathrm{C}$ by injecting a predefined quantity of tert-butyl peroxide (TBP) as a $14.0 \mathrm{wt} \%$ aqueous solution to a jacketed, though uncooled, reactor. One minute later, the semibatch addition of the meta bisulfite (MBS) began, with 1.00 wt \% MBS solutions added drop wise so that the temperature increased at a nominal rate of $0.7^{\circ} \mathrm{C} / \mathrm{min}$. At the peak temperature between 65 and $75^{\circ} \mathrm{C}$ (the exact maximum of the exotherm being comonomer composition dependent), additional quantities of TBP and MBS were added to scavenge residual monomer below $500 \mathrm{ppm}$. The details of the HPLC method for monomer detection have been presented elsewhere. ${ }^{29}$ After the batch had cooled down to $32^{\circ} \mathrm{C}$, wetting agent (Rolfor, TR/8, Cesalpina Chemicals, Italy) was added. The latter allowed for rapid inversion of the flocculant when added to water.

Methylene-bis-acrylamide (MBA) was prepared as $0.1 \mathrm{wt} \%$ solution and added dropwise by a syringe pump. The addition of the MBA began at the outset of the exothermic reaction and was terminated 15 min after the peak temperature was reached. In this way, the addition of MBA was approximately equal as a function of the AM conversion.

AM solutions, at 50 wt \%, and solid MBA were purchased from the former Cytec (now Kemira, Bradford, UK) and used as received. Dimethylaminoethylacrylate methyl chloride (DMAEA) was purchased from Ciba (now BASF, Bradford, UK) and used without purification. Previous studies have indicated that the reactivity ratios are insensitive to the presence of residual copper in AM and hydroquinone (HQ) in the cationic monomer. ${ }^{30}$ The copper was chelated with 167 ppm of EDTA. As chain transfer agents, lactic acid was added at concentration between 0.5 and $1.38 \mathrm{wt} \%$ to control the chain length of the copolymer. The $0.05 \%$ levels of the stabilizing agents (Copper, HQ) added for transport safety had no influence on the molar mass.

The aqueous phase was prepared with deionized water. TBP (purum grade, Fluka, Germany) and sodium MBS (Reactolab, Switzerland) were used as the redox couple. For hydrolytic stability of the polymers, $0.625 \mathrm{wt} \%$ of adipic acid was used directly. Deionized water was added so that the total monomer level of the initial emulsion was $40.0 \mathrm{wt} \%$.

Sorbitan monooleate (Montane 80) and polyoxyethylene sorbitan trioleate (Montanox 85) both (Seppic, France) in an unbleached form with no detectible radical scavenging impurities were blended to obtain a hydrophilic-lipophilic balance (HLB) of 6.0. The total surfactant concentration in the polymerization was $2.4 \mathrm{wt} \%$. Exxsol D100, a narrow cut isoparaffinic mixture, was purchased from Esso.

\section{Materials}

Industrial Precipitated Calcium Carbonate (PCC), schalenoedrical type, was used as standard inorganic substrate in all flocculation experiments. The PCC powder was dried before use. PCC suspensions were prepared at $1 \mathrm{wt} \%$ in distilled water and, to obtain a good dispersion of the particles, the suspension was firstly agitated using magnetic stirring at $600 \mathrm{rpm}$ during $40 \mathrm{~min}$ and then submitted to sonication at $50 \mathrm{kHz}$ during $15 \mathrm{~min}$. The $\mathrm{pH}$ of the PCC suspension was 7.5 and the median of the size distribution of the particles was $0.5 \mu \mathrm{m}$.

The flocculants used were all cationic AM-coDMAEA of high molar mass synthesized as described earlier. The molar mass was kept approximately constant at $5.0 \times 10^{6} \mathrm{~g} / \mathrm{mol}^{3}{ }^{3}$ Copolymer series of three different charge densities have been obtained by varying the weight percentage of the cationic comonomer DMAEA in the monomer feed as indicated in Table I. Further, the appropriate molar composition, charge equivalent, and calculated average linear charge distance are listed in this Table. For the 50 and $20 \mathrm{wt} \%$ compositions, copolymers with three different chain architectures were considered: linear, slightly branched $(+)$, and highly branched $(++++)$. In the case of the $80 \mathrm{wt} \%$ DMAEA, only the linear copolymer was studied. The number of + signs refers to the amount of cross-linker added during the synthesis, $(+)$ equal to 


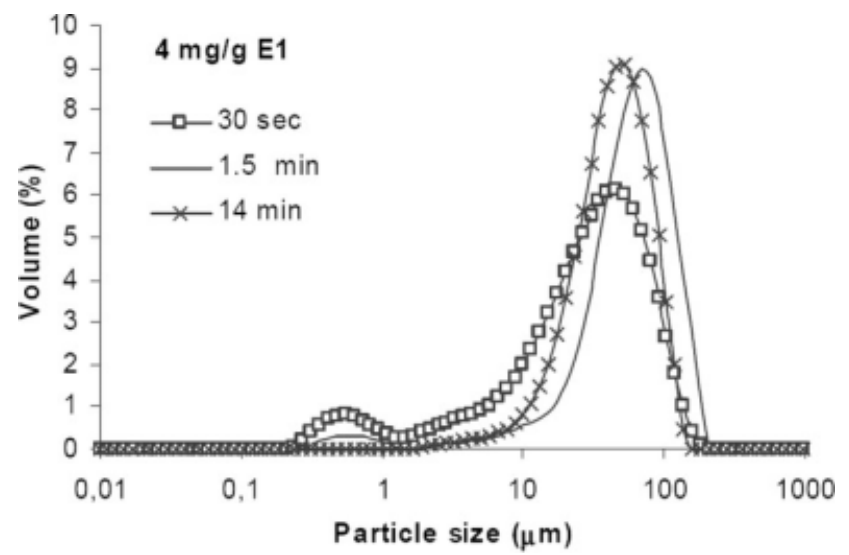

Figure 1 Particle size distribution (volume \% vs. equivalent particle diameter) for flocculation with optimal dosage of E1 monitored at three different time points.

1 aliquot, $(++++)$ equal to 4 aliquots. Here, one aliquot corresponded to $4 \mathrm{ppm}$. This amount was optimized to create an average of one branch per chain.

The stock solutions of the PEL with $40 \mathrm{wt} \%$ copolymer were diluted daily in distilled water to yield solutions of $0.1 \mathrm{wt} \%$. The water conductivity was continuously controlled and kept between 5 to $8 \mu \mathrm{S} /$ $\mathrm{cm}$ to prevent variations in the flocculation process.

\section{Methods}

\section{Polymer characterization}

The IV of the isolated and redissolved copolymers was determined in $0.05 \mathrm{M} \mathrm{NaCl}$ aqueous solution at $20^{\circ} \mathrm{C} \pm 0.1^{\circ} \mathrm{C}$ by dilution viscometry, using an automatic capillary viscometer Viscologic TI1 (Sematech, France), capillary $0.58 \mathrm{~mm}$. The extrapolation to zero concentration was performed according to Huggins as described recently. ${ }^{2}$

\section{PCC flocculation}

PCC flocculation was monitored by measuring the aggregate size by LDS using a Malvern Mastersizer 2000 (Malvern Instruments, UK). The PCC suspension was added to $700 \mathrm{~mL}$ of distilled water in the equipment dispersion unit until $70 \%$ obscuration (average PCC concentration around $0.05 \mathrm{wt} \%$ ), and the tests were carried out setting the pump speed to $1400 \mathrm{rpm}\left(663 \mathrm{~s}^{-1}\right)$. Obscuration was always kept above $5 \%$ to assure a good signal quality. ${ }^{23,24}$ Ideally, obscuration should be $\sim 20 \%$. However, as obscuration decreases during the flocculation test due to floc growth, we have decided to initiate the tests with an obscuration of $70 \%$ to guarantee that obscuration remains higher than $5 \%$ at the end of flocculation.

All flocculants were evaluated over a range of concentrations close to the optimum dosage. A pre- liminary evaluation of the optimum dosage was performed according to Blanco's method ${ }^{16}$ and was then refined by conducting flocculation tests with different PEL concentrations as will be described later. The optimum PEL concentration is the one that leads to larger flocs at the end of the process.

The particle size of PCC was always measured before adding the flocculant to the suspension. Subsequently, a fixed predetermined amount of flocculant was added in one rapid injection to the suspension, and the floc size distribution was measured every minute during $14 \mathrm{~min}$, i.e., until the floc size stabilized. Moreover, the zeta potential of the suspension before and after the flocculant addition was also measured using a Zetasizer Nano ZS (Malvern Instruments UK).

The LDS technique permitted monitoring the mass fractal dimension of flocs during the tests. ${ }^{24,28}$ From the scattering matrix we determined, simultaneously, the fractal dimension coefficient, $d_{F}{ }^{26}$ and the scattering exponent, $\mathrm{SE} .{ }^{27} d_{F}$ is related to the fractal structure of the primary aggregates and SE to the structure of the secondary aggregates. Both are important in floc formation and reorganization under shear, as will be shown herein.

The floc resistance was estimated by submitting the flocs to mechanical shearing caused by sonication. Two different frequencies have been applied over $30 \mathrm{~s}$ periods: $10 \mathrm{kHz}$ and $20 \mathrm{kHz}$. This mechanical shear force was directly applied to the suspension in the LDS dispersion unit after flocculation. After the shearing tests, the shear force was restored to the initial value to allow reflocculation, which was then monitored during another $5 \mathrm{~min}$. Herein, only results for the breakage with the highest frequency $(20 \mathrm{kHz})$ for which breakage is more notorious will be reported.

Monitoring of the flocculation process every $30 \mathrm{~s}$ yields the particle size distribution (PSD) of the

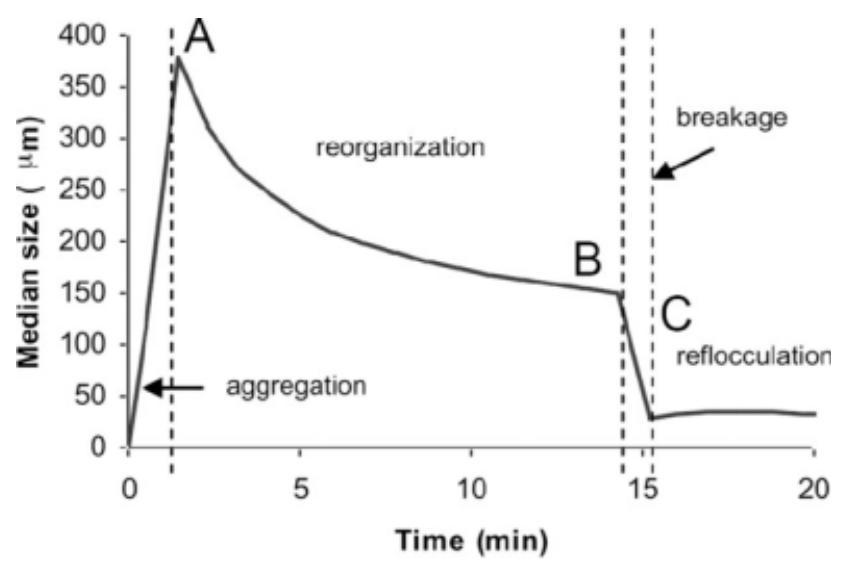

Figure 2 Typical kinetic curve indicating the four phases of the flocculation process: aggregation, reorganization, breakage after sonication, and subsequent reflocculation. $\mathrm{A}, \mathrm{B}$, and $\mathrm{C}$ characterize the median size at transition. 

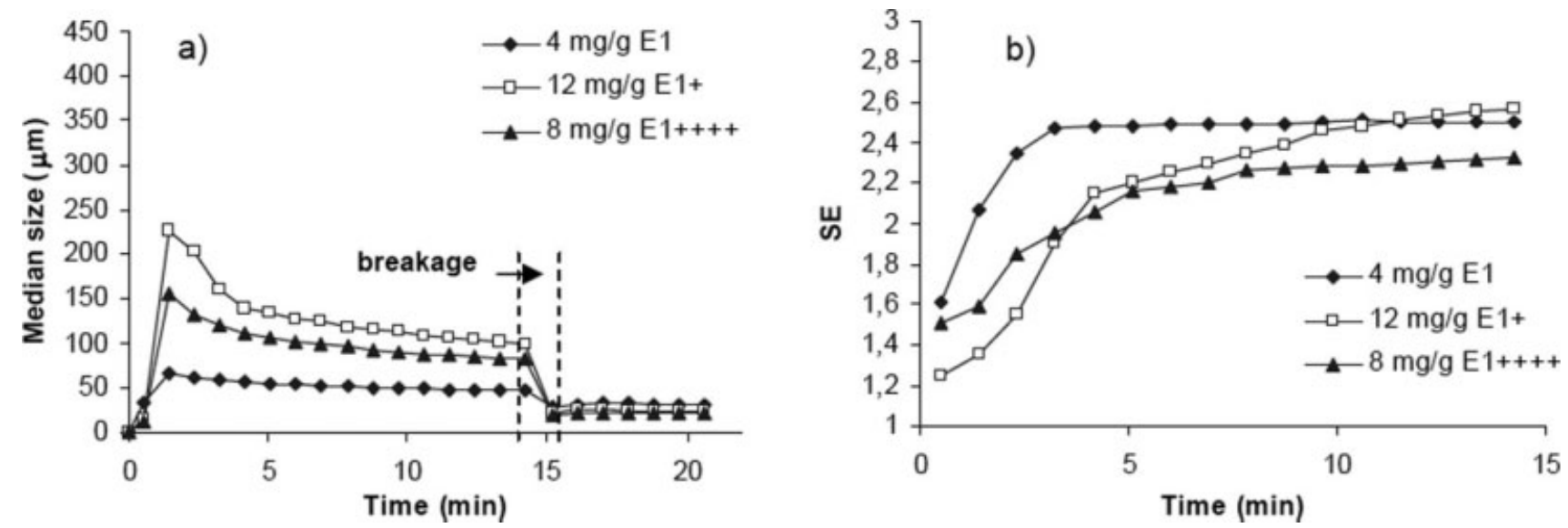

Figure 3 Kinetic curves ${ }^{25}$ (a) and scattering exponent (SE) evolution (b) for the flocculation of PCC with PEL of the E1 series obtained for the previously determined optimum PEL dosage. Breakage is with ultrasound (20 kHz) between 14 and $15 \mathrm{~min}$.

aggregates, as shown exemplary in Figure 1 for flocculation with E1. It is obvious from Figure 1 that just $30 \mathrm{~s}$ after having initiated the flocculation process the primary PCC particles are still present in the suspension, whereas after 1.5 min coinciding with the maximum in the kinetic curve in Figure 2, aggregation was complete. Subsequently, the floc size decreases due to reorganization/equilibration of the aggregates, as can be observed for the PSD at 14 min.

From the PSD at each instant, the kinetic curve for the flocculation process was obtained. Plotting either the median or the average diameter of the flocs as a function of time, a kinetic curve of the type shown in Figure 2 can be constructed.

Four different regions are clearly visible designated as aggregation, reorganization, breakage, and reflocculation region. Moreover, three distinct points are marked with $\mathrm{A}, \mathrm{B}$, and $\mathrm{C}$. The ratios of their median size values B : A and C : B yield, for each kinetic curve, the degree of reorganization and the degree of breakage, respectively, as the relative variation of the floc size.

\section{RESULTS AND DISCUSSION}

\section{Flocculation process}

Following the experimental methodology developed previously by the authors, ${ }^{24,27}$ the influence of the polymer characteristics on the flocculation process as such is presented in Figure 3 for the E1 series $(50 \mathrm{wt}$ $\%$ DMAEA) and in Figure 4 for the G1 series (20 wt $\%$ DMAEA). The figures show the kinetic curves for different polymer dosages around the previously determined optimum and the evolution of the SE with time. The kinetic curve includes also the breakage due to mechanical shearing caused by ultrasound at $20 \mathrm{kHz}$ and the reflocculation stage.

The influence of the chain architecture on the kinetic curve is clearly visible. Moreover, it has an
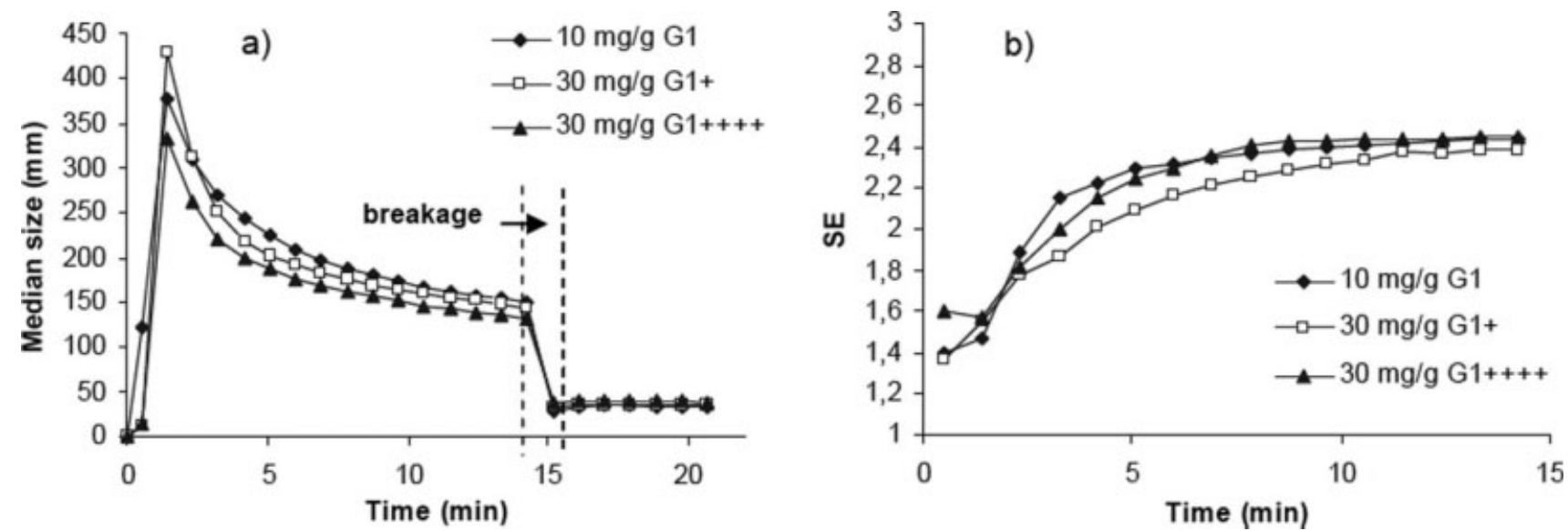

Figure 4 Kinetic curves (a) and scattering exponent (SE) evolution (b) for the flocculation of PCC with PEL of the G1 series obtained for the previously determined optimum PEL dosage. Breakage is with ultrasound (20 kHz) between 14 and $15 \mathrm{~min}$. 
TABLE II

Summary of Parameters Influencing the Flocculation Process

\begin{tabular}{lcl}
\hline \multicolumn{2}{c}{ Predetermined parameters } & \multicolumn{1}{c}{ From flocculation study } \\
\hline $\begin{array}{l}\text { Charge density } \\
\text { Chain archtitecture }\end{array}$ & $\begin{array}{c}\text { Optimum dosage } \\
\text { Zeta potential of the } \\
\text { initial suspension }\end{array}$ & $\begin{array}{l}\text { Floc size at the maximum, } d_{50} \\
\text { Scattering exponent, SE }\end{array}$ \\
Intrinsic viscosity, IV & & $\begin{array}{l}\text { Fractal dimension coefficient, } d_{F} \\
\text { Degree of reorganization, B : A } \\
\end{array}$ \\
& $\begin{array}{l}\text { Degree of breakage, C : B } \\
\text { Zeta potential after PEL addition }\end{array}$ \\
&
\end{tabular}

impact on the floc resistance, percentage of breakage under shear, and reflocculation ability. Specifically, the linear PEL (E1, G1) lead to more resistant flocs. Aggregate restructuring is less for the linear PEL for which smaller flocs were formed because of a lower degree of polymer reconformation. The linear molecules tend to acquire a flatter conformation on the particle surface which leads to higher degree of coverage. ${ }^{9,13,14,19,24,25}$ As a consequence, lower concentration is required and reconformation of the polymer chain and reorganization of the particles in the aggregates are more difficult.

These trends are altogether more evident in the case of higher charge density (E1 series). In the case of the G1 series, the influence of branching is not so pronounced.

Although bridging is probably the main flocculation mechanism, ${ }^{9-11,24,25}$ the lower the charge density the more extended will be the polymer chain on the particle surface because of the lower number of sites available for adsorption in the molecule, leading to larger and more open flocs. ${ }^{24,25,28}$ Details of the underlying flocculation mechanisms have been discussed more extensively previously. ${ }^{25,28}$

Regarding the structure of the flocs, which is evaluated by the SE, the influence of the degree of branch- ing on the compactness of the flocs is evident. The linear polymers (E1 and G1) lead always to more compact flocs (higher SE). In fact, in the case of the linear PEL, they tend to adsorb on the particle surface in a flatter conformation thus resulting in less porous flocs, as discussed elsewhere. ${ }^{28}$ These differences are more visible for the higher charge density [E1 series, Fig. 3(b)]. Indeed, the flocs obtained at the end of flocculation, with G1 of lower charge density, are always less compact [compare Figs. 3(b) and 4(b)]. It is further interesting to analyze the evolution of SE with time, for the different polymers. During the first two min of flocculation, which correspond to reaching the maximum in the kinetic curve, the SE increases from a very low value to a value of around 2 or 2.5. In the case of the branched polymers, SE still increases after that point, due to polymer reconformation. At the end of flocculation, SE is always close to 2.5.

\section{Correlating polymer characteristics with performance in flocculation}

Table II provides a general overview of the parameters which influence the flocculation process. Figures 5-11 present the correlations obtained when experimental values of these parameters were plotted.
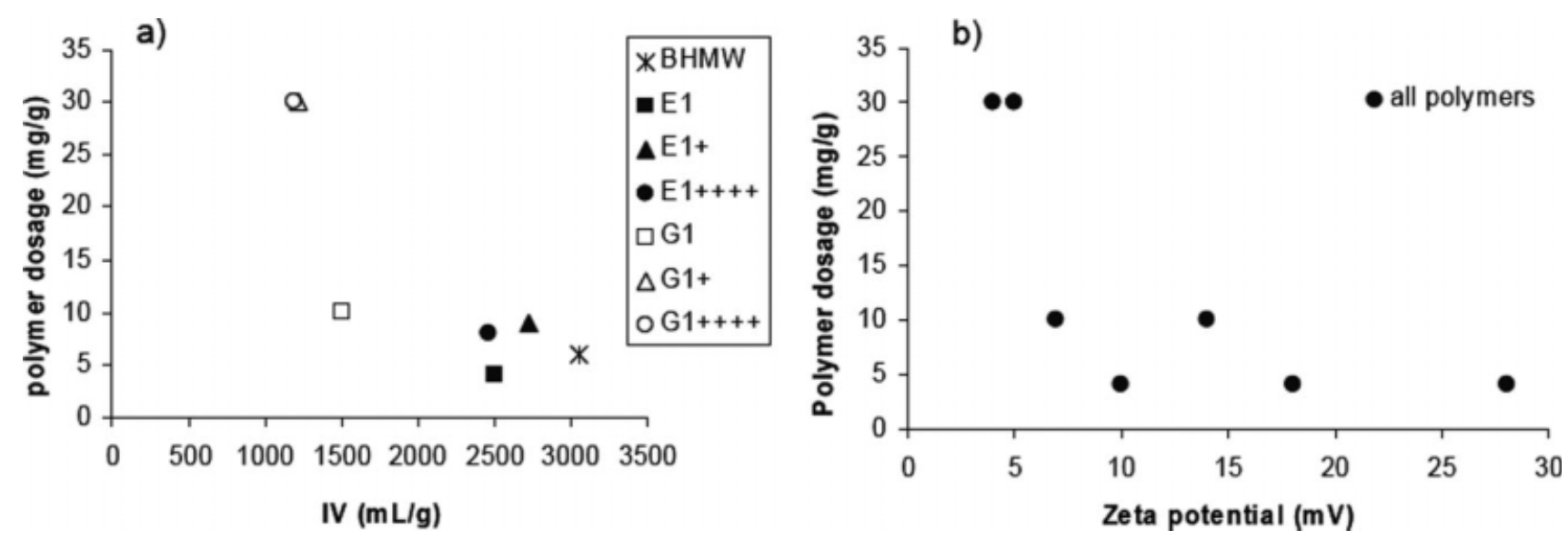

Figure 5 Optimum polymer dosage, mg PEL/g PCC, versus (a) intrinsic viscosity (IV) and (b) zeta potential after flocculant addition for all PEL tested. 


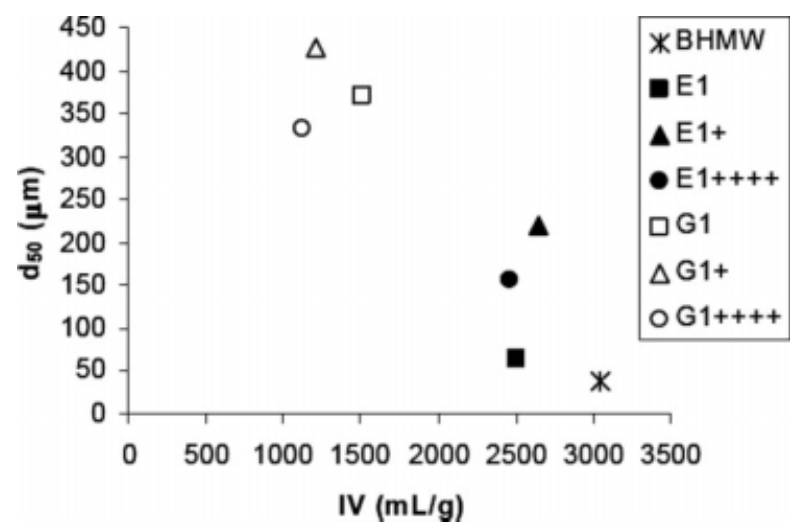

Figure 6 Floc size for the maximum in the kinetic curve $\left(d_{50}\right)$ obtained for the optimum PEL dosages as a function of the intrinsic viscosity (IV).

Figure 5 shows the dependence of the optimum polymer dosage on the intrinsic IV of the PEL in $0.05 \mathrm{M} \mathrm{NaCl}$ and zeta potential of the suspension immediately following the addition of polymer. For the seven PEL evaluated, the optimum dosages were in the range of $4-30 \mathrm{mg}$ of PEL/g of PCC. Higher IV corresponds to larger hydrodynamic dimensions of the polymer coils of similar molar mass. Therefore, as IV increases a higher degree of coverage of the particles surface can be obtained and a lower dosage will be required.

Correlation with the IV instead of the molar mass appeared to be more suitable in this study. For the same molar mass, the hydrodynamic dimensions are different when the chain architecture and the charge density vary. A more detailed macromolecular characterization of the samples $\mathrm{E} 1++++$ and $\mathrm{G} 1++++$ was reported previously. ${ }^{2}$ Despite the different IVs, the molar mass analyzed by analytical ultracentrifugation was similar, $5.3 \times 10^{6} \mathrm{~g} / \mathrm{mol}$ for $\mathrm{E} 1++++$ and $4.6 \times 10^{6} \mathrm{~g} / \mathrm{mol}$ for $\mathrm{G} 1++++$, all characterizations performed in $0.05 \mathrm{M} \mathrm{NaCl}$ at $20^{\circ} \mathrm{C}$. The interdependencies of the hydrodynamic dimensions, the molar mass and chain architecture are in particular complicated for PEL and not a priori predictable. Therefore, the IV as a rigorous parameter was used here.

As for the dependency on the zeta potential of the suspension, the main factor influencing the zeta potential is the charge density of the PEL. In all the tests, we have started with a suspension of fixed zeta potential $(\sim-27 \mathrm{mV})$; thus, the highest values of zeta potential after flocculant addition refer to the polymers with higher charge density. Therefore, the value of the zeta potential after flocculant addition can give a good indication of the level of polymer required for the flocculation process.

In Figures 6 and 7, we correlated the floc characteristics with the IV and the zeta potential. Referring to the floc size, represented by its median diameter $d_{50}$ (Fig. 6), it is evident that the floc size decreases when the IV increases. The results plotted refer to the size at the maximum in the kinetic curve, as PEL are often applied in very dynamic environments where flocculation occurs within seconds-to-minutes. A similar trend is observed if the floc size at the end of flocculation is considered. However, the variation is less pronounced.
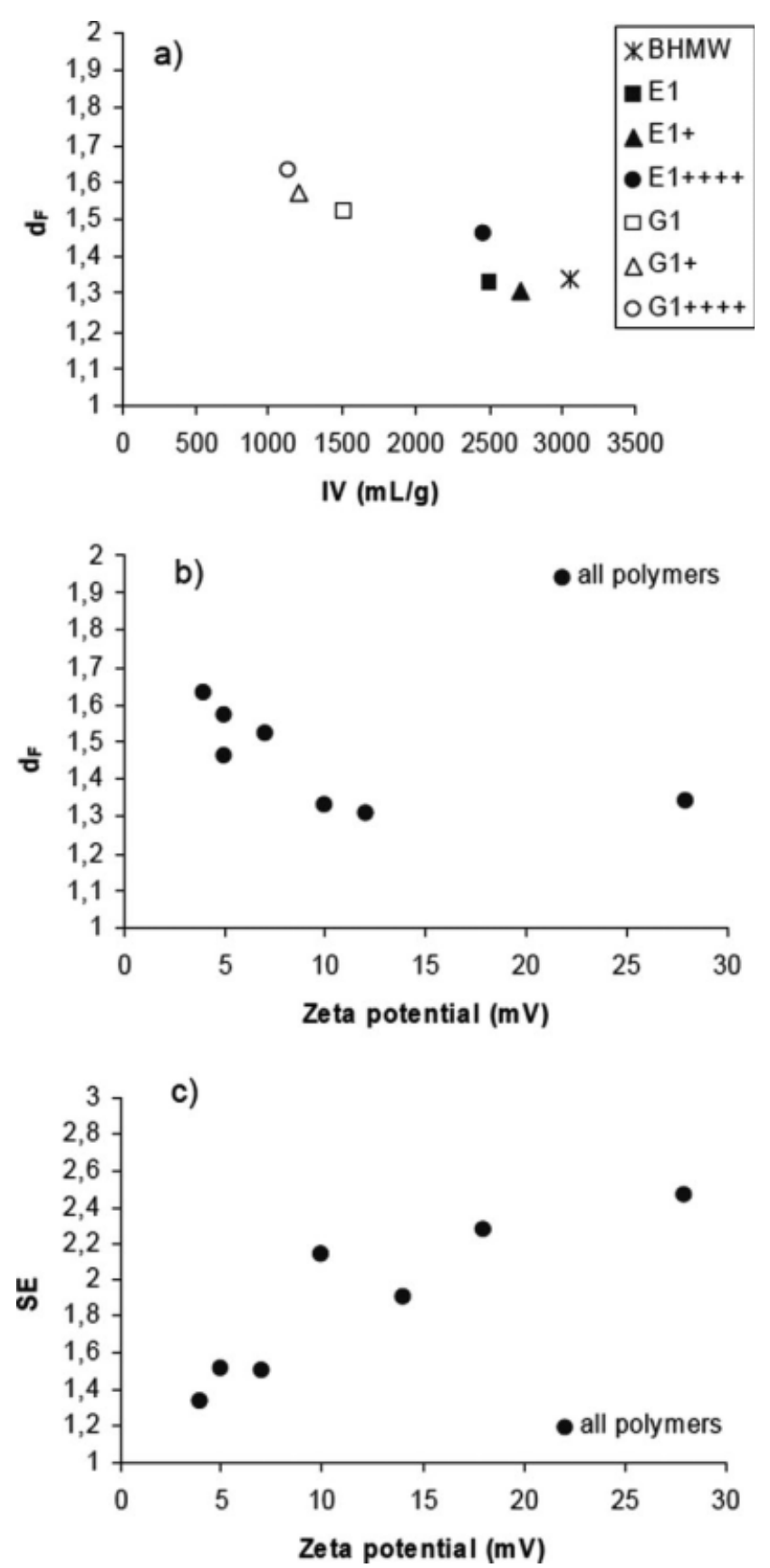

Figure 7 Fractal dimension $\left(d_{F}\right)$ ( $\mathrm{a}$ and $\mathrm{b}$ ) and scattering exponent (SE) (c) for the maximum in the kinetic curve and for the optimum polymer dosage versus intrinsic viscosity (IV) and zeta potential after flocculant addition. 

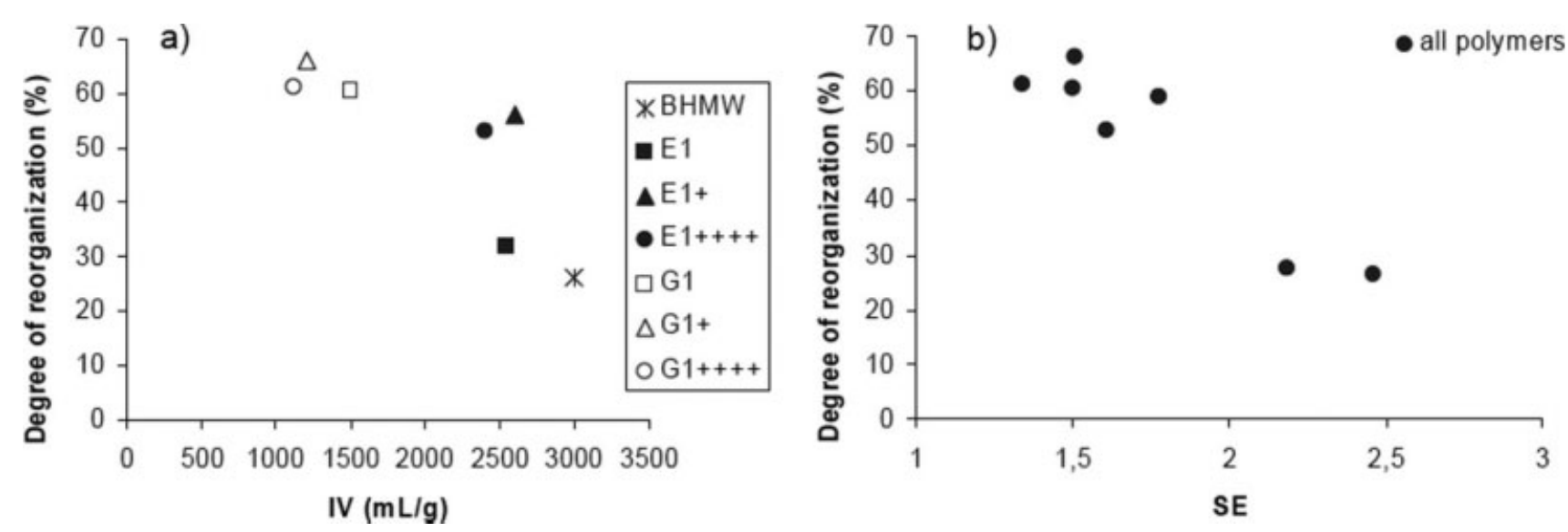

Figure 8 Degree of floc reorganization for the optimum dosage versus intrinsic viscosity IV (a) and scattering exponent (SE) (b) for all PEL tested.

Further, it is meaningful to correlate the floc structure with the IV and the zeta potential of the suspension measured after the addition of the flocculant (Fig. 7). Here, the $d_{F}$ and SE for the maximum in the kinetic curve are plotted versus IV and the zeta potential, since, as noted previously, these parameters are essentially invariant at the end of flocculation. Specifically, neither the polymer type or concentration influence the $d_{F}$ or SE values when the flocculation nears completion. ${ }^{25}$

In Figure 7(a), the fractal dimension decreases as IV increases. This agrees with the fact that higher IV corresponds to more extended polymer coils. Thus, more open primary flocs with a lower $d_{F}$ result.

Referring to the influence of the zeta potential on $d_{F}$ [Fig. 7(b)], the fractal dimension tends to decrease as the zeta potential increases. This must be related with the fact that for PEL with a higher charge density, which lead to suspensions with a higher zeta potential, flocculation is faster and, thus, the primary aggregates described by $d_{F}$ do not have enough time to form a very compact structure. The opposite happens with the secondary aggregates, whose structure has to be described by the scattering exponent SE [Fig. 7(c)]. In fact, if flocculation occurs faster, a larger number of secondary aggregates already exist at the maximum of the kinetic curve, which are, simultaneously, already more structured.

It is also possible to correlate the level of reorganization in the kinetic curve with the polymer characteristics. The results in Figure 8(a), though presenting some variation, show a general trend of lower degree of reorganization as the IV increases. As discussed earlier, again larger coil volume accompanied by less free space on the particle surface for the polymer to reconform can serve as explanation. However, the main correlation is between the degree of reorganization and the scattering exponent at the maximum in the kinetic curve. Reorganization occurs mainly among the secondary aggregates. This explains the good correlation with SE, which is related to the structure of those aggregates [Fig.
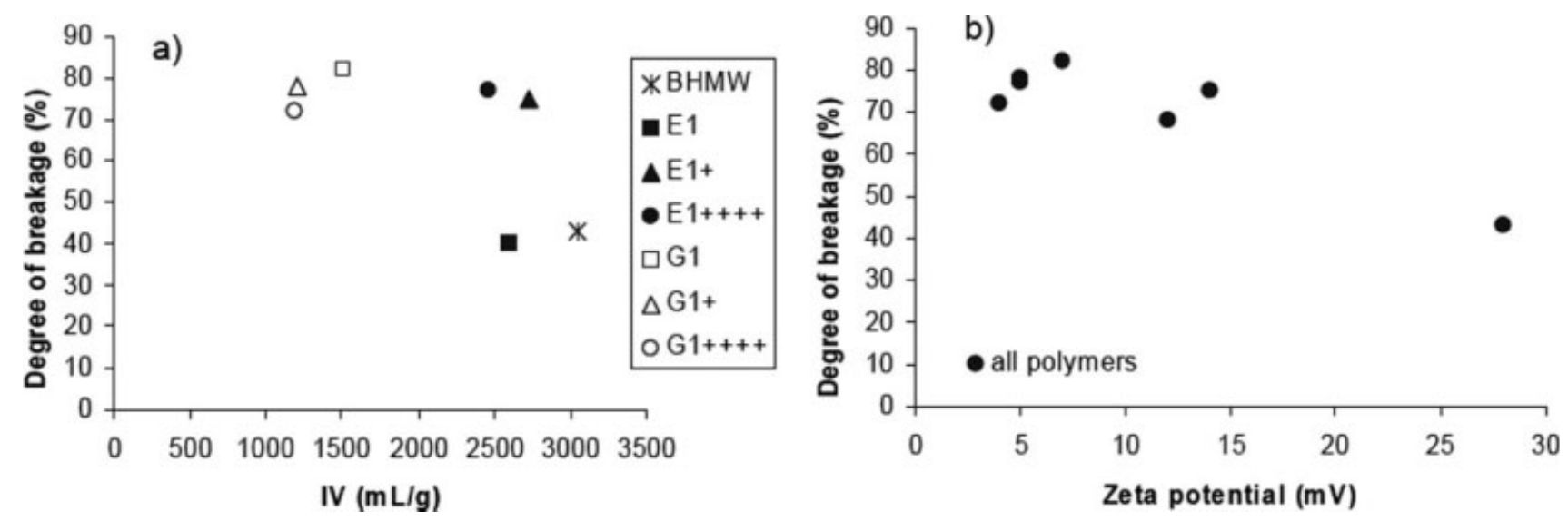

Figure 9 Degree of breakage for the optimum dosage versus intrinsic viscosity IV (a) and zeta potential after flocculant addition (b) for all PEL tested. 

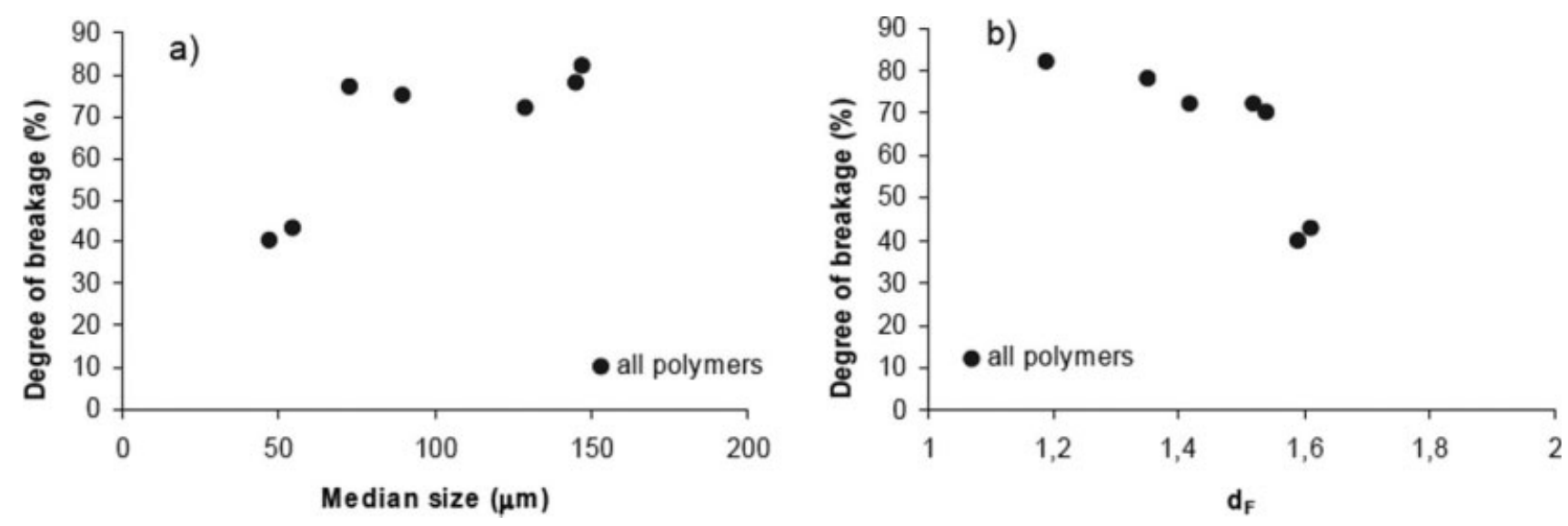

Figure 10 Degree of breakage versus floc characteristics (a) median size and (b) fractal dimension $\left(d_{F}\right)$ for optimum dosage for all PEL tested.

8(b)]. The larger SE, the more compact are the flocs, and the lower is the degree of reorganization, as expected.

Examining how the resistance of the flocs depends on the IV and the zeta potential in Figure 9, no clear trend can be concluded. Apparently, other factors rather than IV affect the resistance of the flocs. Indeed, the polymer chain architecture is likely playing an important role in flocs resistance. It is known that linear polymers lead to stronger flocs. Contrarily, the percentage breakage (inverse of flocs resistance) can be clearly correlated with the zeta potential of the suspension [Fig. 9(b)]. Higher zeta potential, resulting from polymers with a higher charge density, yields stronger flocs (lower degree of breakage) as already observed in a previous study. ${ }^{24}$

Finally, the resistance of the flocs depends on the floc size and structure, fractal dimension, at the end of the flocculation process, as visible in Figure 10. Larger flocs break more easily and more open flocs (smaller $d_{F}$ ) are less resistant. In addition, Figure 11 presents the influence of the polymer dosage on the floc resistance for the low charged G1 series. It is evident that there is a maximum in the breakage versus concentration curve for all three G1 polymers. This maximum coincides with the optimum flocculant dosage for each polymer. Again noteworthy, the floc size is most important for the flocs resistance, as the optimum polymer dosage has been defined as the one leading to larger flocs. When the polymer is added in excess, concentrations above the optimum, the flocs become more resistant. In practice, even if the flocs break as a result of the mechanical action, they reflocculate immediately due to the excess of the PEL. More branched polymers require a higher dosage. However, they have been shown to produce tighter flocs, ${ }^{28}$ and this correlates with improved shear resistance.

\section{CONCLUSIONS}

In this study, it was observed that floc size, resistance, and reorganization are correlated with the IV of the cationic copolymers and the zeta potential of suspensions (see Table III). Lower IVs, which are, in this study, associated with the PEL of lower charge density, yielded larger flocs, which are less resistant. Concomitantly, flocs with lower scattering exponents, associated with the fractal nature of the secondary aggregates, are more fragile. The zeta potential of the suspension, just after flocculant addition, correlates well with the floc structure and resistance. Indeed, if the particle characteristics are kept constant, this parameter, closely related with the PEL charge density, can provide important insight about the probable floc properties.

Thus, based on the correlations obtained, it can be anticipated that if high floc resistance is required, even if only low flocculation times are allowed as

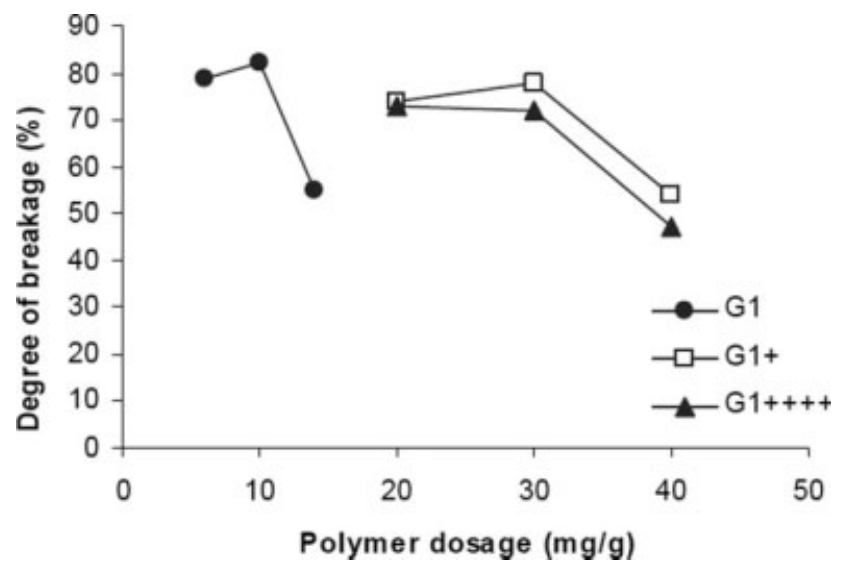

Figure 11 Degree of breakage versus PEL dosage, mg PEL/g PCC, for the G1 series. 
TABLE III

Effect of Polymer Characteristics on Flocculation Efficiency

\begin{tabular}{|c|c|c|c|c|c|}
\hline $\begin{array}{c}\text { Polymer } \\
\text { characteristics }\end{array}$ & $d_{50}(\mu \mathrm{m})$ & $\begin{array}{c}\text { Degree of } \\
\text { reorganization }(\%)\end{array}$ & $\begin{array}{c}d_{F}(\max . \\
\text { kinetic curves })\end{array}$ & $\begin{array}{c}\text { SE (max. } \\
\text { kinetic curves) }\end{array}$ & $\begin{array}{c}\text { Degree of } \\
\text { breakage }(\%)\end{array}$ \\
\hline $\begin{array}{l}\text { Intrinsic Viscosity } \\
\text { Zeta potential } \\
\text { Optimum dosage }\end{array}$ & Strongly dependent & Strongly dependent & $\begin{array}{l}\text { Correlates } \\
\text { Correlates }\end{array}$ & $\begin{array}{l}\text { Correlates } \\
\text { Correlates }\end{array}$ & $\begin{array}{l}\text { Correlates } \\
\text { Correlates } \\
\text { Maximum }\end{array}$ \\
\hline
\end{tabular}

for instance in paper making, PEL with high IV should be selected. These are usually branched polymers of high molar masses, which can have medium charge density as in the E1 series tested in this study. If, on the other hand, high flocculation times can be allowed and resistance is not a key parameter, one can aim at flocs that reform to denser structures during flocculation, even if that requires longer flocculation times. PEL of lower charge density, as in the case of the G1 series in this study, can then be used.

Overall, floc properties are strongly dependent, as expected, on the polymer characteristics. Thus, any step forward to the establishment of a qualitative or quantitative correlation between suitable parameters, as done in this study, is considered as important for the easier selection of a flocculant for a specific application. The use of both model particles and a model suspending medium to better understand these interrelations is a first step.

\section{References}

1. Field, J. R.; Farrar, D.; Flesher, P. U.S. Pat. 4,820,645 (1989).

2. Bourdillon, L.; Hunkeler, D.; Wandrey, C. Prog Colloid Polym Sci 2006, 131, 141.

3. Hernandez Barajas, J.; Wandrey, C.; Hunkeler, D. Polym News 2004, 29, 239.

4. Hubbe, M. A. Paper Technol 2004, 45, 8.

5. Carnal, F.; Laguecir, A.; Stoll, S. Colloid Polym Sci 2004, 283, 317.

6. Hernandez Barajas, J.; Wandrey, C.; Hunkeler, D. J. U.S. Pat. 6,667,374 B1 (2003).

7. Hernandez Barajas, J.; Wandrey, C.; Hunkeler, D. J. U.S. Pat. 6,617,402 B2 (2003).

8. Hernandez Barajas, J.; Wandrey, C.; Hunkeler, D. J. U.S. Pat. 6,294,622 B1 (2001).
9. Gregory, J. Proceedings of Sedimentation and Consolidation, Engineering Foundation Conference, Georgia, USA, 1985.

10. Eklund, D.; Lindström, T. Paper Chemistry: An Introduction; DT Paper Science Publications: Finland, 1991.

11. Berlin, A.dA.; Kislenko, V. N. Colloid Surf 1995, 104, 67.

12. Bremmell, K. E.; Jameson, G. J.; Biggs, S. Colloid Surf 1998, 139, 199.

13. Biggs, S.; Habgood, M.; Jameson, G. J.; Yan, Y. D. Chem Eng J 2000, 80, 13.

14. Cadotte, M.; Tellier, M. E.; Blanco, A.; Fuente, E.; Van De Ven, T. G. M.; Paris, J. Can J Chem Eng 2007, 85, 240.

15. Hubbe, M.; Nanko, N.; Mcneal, M. R. Bioresources 2009, 4, 850.

16. Blanco, A.; Negro, C.; Hooimijer, J.; Tijero, J.; Appita, J. 1996, 49, 113.

17. La Mer, V. K.; Healy, T. W. Rev Pure Appl Chem 1963, 13, 112.

18. Fleer, G. J.; Scheutjens, J. M. H. M. In Coagulation and Flocculation: Theory and Applications; Dobias, B., Ed. Marcel Dekker: New York, 1993; Chapter 5.

19. Blanco, A.; Negro, C.; Fuente, E.; Tijero, J. Ind Eng Chem Res 2005, 44, 9105.

20. Blanco, A.; Fuente, E.; Negro, C.; Tijero, J. Can J Chem Eng 2002, 80, 734 .

21. Swerin, A.; Risinger, L.; Ödberg, L. J Pulp Paper Sci 1997, 23, 374.

22. van de Ven, T. G. M.; Alince, B. J. Colloid Interf Sci 1996, 181, 73.

23. Rasteiro, M. G.; Garcia, F. A. P.; Perez, M. Part Sci Tech 2007, $25,303$.

24. Rasteiro, M. G.; Garcia, F. A. P.; Ferreira, P.; Blanco, A.; Negro, C.; Antunes, E. Powder Technol 2008, 183, 231.

25. Antunes, E.; Garcia, F. A. P.; Ferreira, P.; Blanco, A.; Negro, C.; Rasteiro, M. G. Ind Eng Chem Res 2008, 47, 6006.

26. Teixeira, J. J Appl Cryst 1988, 21, 781.

27. Liao, J. Y. H.; Selomulya, C.; Bushell, G.; Bickert, G.; Amal, R. Part Part Syst Charact 2005, 22, 299.

28. Rasteiro, M. G.; Garcia, F. A. P.; Ferreira, P.; Blanco, A.; Negro, C.; Antunes, E. Chem Eng Proc 2008, 47, 1329.

29. Hernandez Barajas, J.; Hunkeler, D.; Petro, M. J Appl Polym Sci 1996, 61, 1325.

30. Hunkeler, D.; Hamielec, A. E. Polymer 1991, 32, 2626. 